\title{
Resección transanal de un adenoma velloso rectal a través de un trocar monopuerto*
}

\author{
Drs. FRANCISCO LÓPEZ K. ${ }^{1}, \mathrm{M}^{\mathrm{a}}$ ISABEL QUIJADA G. ${ }^{1}$, CRISTÓBAL SUAZO L. ${ }^{\text {, }}$ \\ UDO KRONBERG ${ }^{1}$, ALEJANDRO ZÁRATE I. $^{1}$ \\ 1 Unidad de Coloproctología Clínica Las Condes. \\ Santiago, Chile.
}

\begin{abstract}
Excision of a rectal villous adenoma using a transanal single port trocar

Single port access for minimally invasive surgery is feasible nowadays. We report a 55 years old female presenting with hematochezia. During a colonoscopy, a villous adenoma of $2 \mathrm{~cm}$ in diameter located $5 \mathrm{~cm}$ above the anal margin, was found. This lesion was excised through the anus, using a single port trocar. The procedure lasted 45 minutes, the postoperative evolution was uneventful and the patient was discharged 24 hours after the procedure.
\end{abstract}

Key words: Transanal surgery, villous adenoma, minimally invasive surgery.

\section{Resumen}

Posterior al advenimiento de la cirugía laparoscópica colorrectal, desde comienzos de la década de los 90, se han desarrollado diversos tipos de accesos mínimamente invasivos, siendo hoy en día el acceso por un puerto único una alternativa factible. Se presenta el caso clínico de una paciente de 55 años, a la cual por sintomatología se le diagnóstica una lesión rectal, la que luego de ser estudiada endoscópica y endosonográficamente, es resecada por monopuerto vía anal. La cirugía duró 45 minutos y no presentó eventos adversos intraoperatorios. Su evolución quirúrgica fue satisfactoria con alta hospitalaria al día siguiente de la cirugía.

Palabras clave: Tumores rectales, cirugía mínimamente invasiva, monopuerto.

\section{Introducción}

La resección local transanal (RLT) permite la extirpación de tumores rectales con una baja morbimortalidad y resultados funcionales satisfactorios. En la actualidad sus mayores indicaciones son pacientes con tumores rectales benignos y el cáncer de recto con compromiso superficial de la pared (tumores con compromiso hasta la submucosa: uT0 uT1). La RLT de tumores más avanzados (uT2-uT3), se asocia a una alta tasa de compromiso ganglionar y de recidiva local, por lo que en esos casos no se aconseja este abordaje salvo en pacientes de alto riesgo quirúrgico ${ }^{1-3}$. Al analizar las distintas series

*Recibido el 18 de noviembre de 2011 y aceptado para publicación el 12 de marzo de 2012.

Los autores no declaran conflictos de interés.

Correspondencia: Dr. Francisco López-Köstner.

Lo Fontecilla 441. Santiago, Chile.

flopez@clc.cl 
publicadas llama la atención la importante variabilidad de los resultados, lo que podría estar condicionado por la dificultad técnica y los márgenes de la resección ${ }^{4,5}$. Debemos señalar que la técnica ideal debiera incorporar una buena dilatación del esfínter anal, el uso de retractores apropiados, logrando así una buena exposición que permita la resección con un margen libre de tumor seguro. A todos estos requerimientos técnicos debemos agregar el hecho que los criterios de selección para realizar esta cirugía son estrictos (Tabla 1), por tanto, en todos los equipos quirúrgicos, la RLT es una intervención infrecuente ${ }^{1,6}$. A inicios de la década de los ochenta se reporta el uso de la resección endoscópica transanal microquirúrgica (TEM) como una medida para disminuir las dificultades técnicas de la RLT y aumentar la distancia a la cual se pueden extirpar los tumores por vía transanal. A la fecha son múltiples los reportes que muestran la seguridad de esta técnica $^{7,8}$, pero su gran inconveniente para una mayor aceptación ha sido su elevado costo ${ }^{3}$.

Con el advenimiento de la cirugía laparoscópica colorrectal desde comienzos de la década de los 90, se han desarrollado diversos tipos de accesos mínimamente invasivos, siendo hoy en día el acceso por un puerto único una alternativa factible $\mathrm{e}^{9-13}$.

Recientemente se han desarrollado diversos dispositivos que permiten el abordaje laparoscópico a través de un solo orificio y se han reportado en la literatura diversos procedimientos realizados por este acceso ${ }^{6,14,15}$.

El objetivo de esta comunicación es presentar un nuevo abordaje transanal mediante un puerto único e instrumental laparoscópico.

\section{Caso clínico}

Se trata de un paciente de 55 años sin comorbilidades, que consulta 3 semanas previo a la cirugía, por cuadro de tres meses de evolución caracterizado por rectorragia ocasional, sin baja de peso ni otros síntomas. Al tacto rectal se palpa una lesión blanda en cara lateral derecha del recto inferior. En la colonoscopia se evidencia un pólipo sésil de aspecto velloso de aproximadamente $2 \mathrm{~cm}$ de diámetro, de base ancha ubicada en la pared lateral derecha del recto bajo sugerente de adenoma velloso (Figura 1). Con el rectoscopio rígido se define que su borde inferior se encuentra a $5 \mathrm{~cm}$ del margen anal.

La endosonografía realizada no evidencia compromiso submucoso ni adenopatías perirrectales (Figura 2). La anatomía patológica confirma el diagnóstico de adenoma túbulo velloso con displasia severa focal. Se plantea para una resección quirúrgica transanal.

\section{Tabla 1. Criterios para resección transanal}

\section{Criterios clínico-endoscópicos:}

a) Hasta $4 \mathrm{~cm}$ de diámetro

b) Hasta $9 \mathrm{~cm}$ del margen anal

c) Menor a $1 / 3$ de la circunferencia rectal

d) No ulcerado, no indurado

e) Móvil al tacto

f) No susceptible de resección endoscópica

Criterios estadificación:

a) EUS: lesión hasta uT1

b) RNM + TAC: $\sin$ metástasis ganglionares ni a distancia

\section{Cirugía}

Luego de inducir una anestesia general, la paciente se coloca en posición de litotomía. Se prepara el periné de manera convencional con povidona yodada, y se realiza un lavado del recto. La profilaxis antibiótica se indica con Acantex-Metronidazol y se coloca una Sonda Foley.

Se introduce un monotrócar Covidien ${ }^{\circledR}$ y se insufla $\mathrm{CO}_{2}$ para neumorrecto con presión máxima de $8 \mathrm{mmHg}$. La visualización laparoscópica se realiza mediante cámara Olympus Endo-Eye ${ }^{\circledR}$, observando la lesión vellosa en su totalidad y sus bordes de manera nítida (Figura 3). Se levanta la lesión inyectando suero fisiológico al espacio submucoso. Luego se diseca con gancho monopolar inicialmente toda la circunferencia de la lesión con una distancia de aprox. $5 \mathrm{~mm}$ sin dificultades (Figura 4). En un sitio queda un margen macroscópico más cercano por lo que se amplía nuevamente la resección. Luego se diseca el plano submucoso de la misma manera, logrando una resección completa, con márgenes macroscópicos negativos (Figura 5). Se controla la hemostasia en forma satisfactoria.

No se sutura la pared ya que la disección se ha llevado a cabo en el plano submucoso. El tiempo quirúrgico fue de $45 \mathrm{~min}$.

La paciente evoluciona en buenas condiciones generales, sin dolor abdominal ni perianal en el postoperatorio. Es dada de alta el día siguiente por su buena evolución, sin rectorragia, ni dolor. Se indicó analgesia según requerimiento y dieta liviana con abundante fibra por vía oral.

La anatomía patológica de la lesión informa un adenoma tubular sésil, con displasia intraepitelial moderada con un margen quirúrgico focalmente en contacto con la lesión que posteriormente fue ampliado.

Los resultados de este caso, sumados a los previamente reportados evidencian que este tipo de procedimientos es factible. 


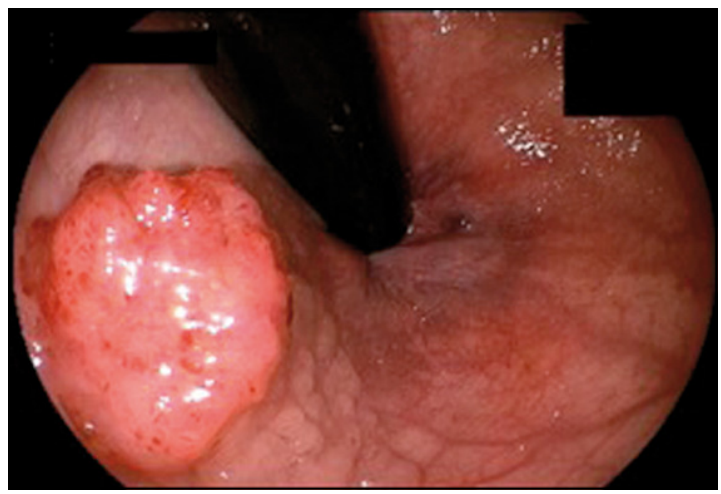

Figura 1. Adenoma rectal retrovisión colonoscópica.

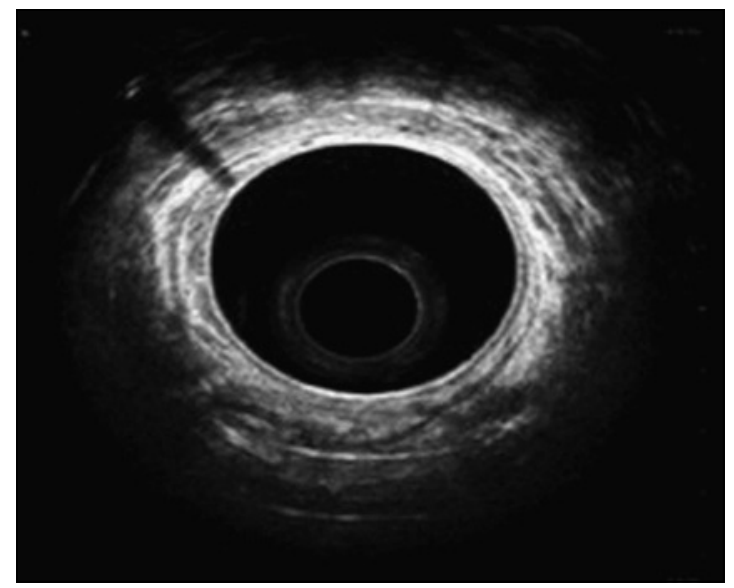

Figura 2. Endosonografía rectal. Lesión a nivel de mucosa, en cara posterolateral derecha en la que impresiona ser un adenoma velloso sin compromiso de submucosa.

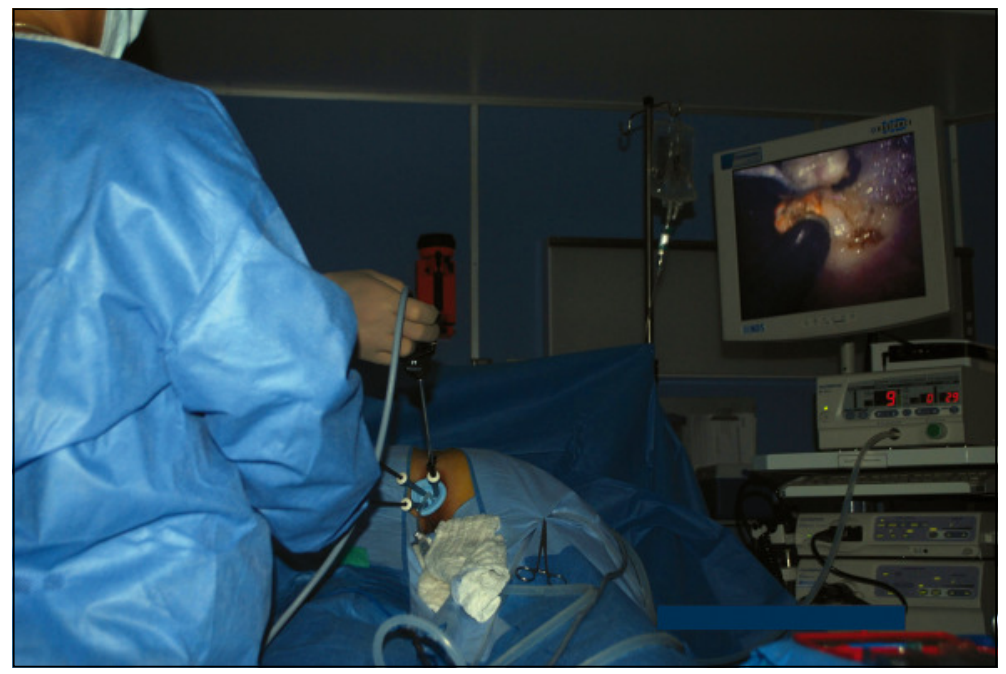

Figura 3. Visión laparoscópica intraoperatoria. Resección con electrobisturí en plano submucoso de la lesión con margen libre.

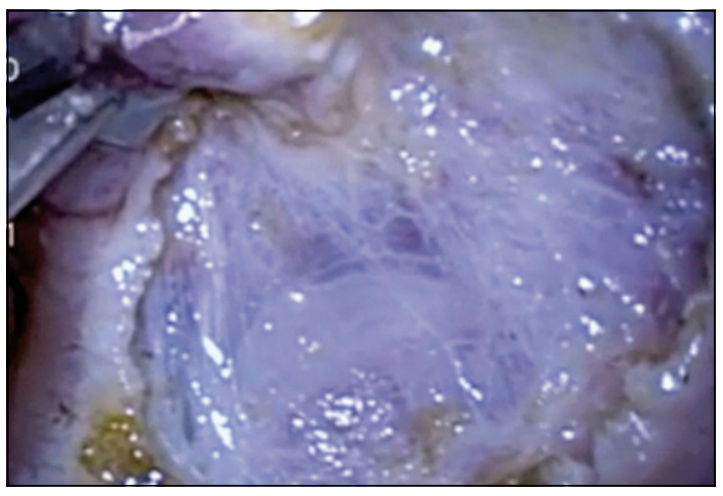

Figura 4. Visión laparoscópica del plano de disección submucosa.

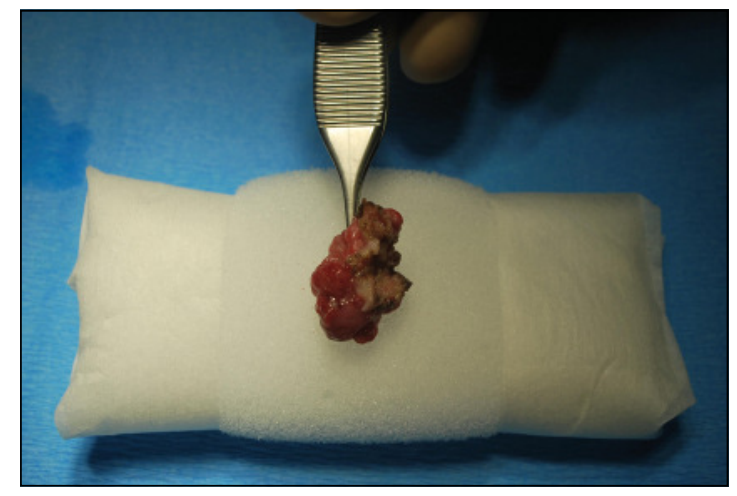

Figura 5. Pieza operatoria completa. 
Hay que destacar que el monotrocar utilizado es un dispositivo flexible, de fácil instalación y extracción, con cuatro orificios para la introducción de trocares e insuflación de $\mathrm{CO}_{2}$ continuo para mantener la presión deseada. Este dispositivo tiene forma de reloj de arena, con un diámetro de $3 \mathrm{~cm}$, por lo que no requiere divulsión del canal anal para su introducción, de esta forma se logra un correcto posicionamiento del instrumento, sin dañar el esfinter anal.

Cabe mencionar que al igual que la RLT clásica, el paciente se beneficia de la mínima invasión del procedimiento, lo que se evidencia en un tiempo quirúrgico corto, bajos requerimientos de analgesia endovenosa en el postoperatorio, y una rápida recuperación con un alta precoz. A estas ventajas se agregan los beneficios propios de la RLT por monotrocar, como la comodidad para el cirujano de trabajo en recto medio-superior, y la excelente visualización de alta resolución, lo que se traduce en una mayor facilidad para la resección de la pieza con márgenes negativos y la extracción de la pieza quirúrgica completa en bloc, lo cual resulta fundamental para su estudio anatomopatológico y aumenta la seguridad oncológica de la resección.

Mediante una protocolización y selección correcta de los pacientes, este tipo de abordaje podría transformarse en el futuro cercano en una alternativa segura y reproducible por grupos quirúrgicos experimentados en técnicas mínimamente invasivas.

\section{Referencias}

1. Peng J, Chen W, Sheng, Xu Y, Cai G, Huang D, Cai S. Oncological outcome of $\mathrm{T} 1$ rectal cancer undergoing standard resection and local excision. Colorrectal Dis. 2011;13:14-9.

2. Cuthbertson AM, Kaye AH. Local excision of carcinomas of the rectum, anus, and anal canal. Aust $\mathrm{N} \mathrm{Z} \mathrm{J}$ Surg. 1978;48:412-5.

3. Horn A, Halvorsen JF, Morild I. Transanal extirpation for early rectal cancer. Dis Colon Rectum 1989;32:76972.
4. Piccinini EE, Ugolini G, Rosati G, Conti A. Transanal local resection for benign and malignant rectal tumours. Int J Colorectal Dis. 1995;10:112-6.

5. Kim E, Hwang J, García-Aguilar J. Local Excision for Rectal Carcinoma. Clinical Colorectal Cancer 2008;7:376-85.

6. Dardamanis D, Theodorou D, Theodoropoulos G, Larentzakis A, Natoudi M, Doulami G, et al. Transanal polypectomy using single incision laparoscopic instruments. World J Gastrointest Surg. 2011;3:56-8.

7. Bretagnol F, Merrie A, George B, Warren BF, Mortensen NJ. Local excision of rectal tumours by transanal endoscopic microsurgery. Br J Surg. 2007;94:627-33.

8. Moore JS, Cataldo PA, Osler T, Hyman NH. Transanal endoscopic microsurgery is more effective than traditional transanal excision for resection of rectal masses. Dis Colon Rectum 2008;51:1026-30.

9. Waters JA, Guzmán MJ, Fajardo AD, Selzer DJ, Wiebke EA, Robb BW, George VV. Single-port laparoscopic right hemicolectomy: a safe alternative to conventional laparoscopy. Dis Colon Rectum 2010;53:1467-72.

10. Gaujoux S, Bretagnol F, Ferron M, Panis Y. Singleincision laparoscopic colonic surgery. Colorectal Dis. 2011;13:1066-71.

11. López-Kostner F, Cápona R, Heine C, Wainstein C, Márquez N. Hemicolectomía derecha laparoscópica por puerto único para cáncer de colon. Rev Chil Cir. 2009;61:566-70.

12. Gaujoux S, Bretagnol F, Ferron M, Panis Y. Singleincision laparoscopic colonic surgery. Colorectal Dis. 2011;13:1066-71.

13. Waters JA, Guzmán MJ, Fajardo AD, Selzer DJ, Wiebke EA, Robb BW, et al. Single-port laparoscopic right hemicolectomy: a safe alternative to conventional laparoscopy. Dis Colon Rectum 2010;53:1467-72.

14. Asencio F, Uribe N, Balciscueta ZR, Rueda C, Ortiz I. Cirugía endoscópica transanal con material convencional de laparoscopia ¿es factible? Cir Esp. 2011; 89: 101-5.

15. Cantero Cid R, García JC, González T, Lima F, Martínez J, Martín R, Torres J. Resección transanal a través de un trocar monopuerto. Una nueva aproximación al NOTES Cir Esp. 2011;89:20-3. 